\title{
Addressing single spin crossover molecules in a two-dimensional monolayer
}

\section{Yongfeng Tong}

SOLEIL-Synchrotron

Massine Kelaï

Université de Paris / CNRS

Kaushik Bairagi

Université de Paris / CNRS

Vincent Repain

Université de Paris / CNRS

Jérôme Lagoute

Université de Paris / CNRS

Yann Girard

Université de Paris / CNRS

Sylvie Rousset

Université de Paris / CNRS

Marie-Laure Boillot

Université Paris-Saclay / CNRS

Talal Mallah

Université Paris-Sud 11

Cristian Enachescu

Alexandru Ioan Cuza University of lasi

Amandine Bellec ( $\nabla$ amandine.bellec@univ-paris-diderot.fr )

Université de Paris / CNRS https://orcid.org/0000-0001-7410-9736

\section{Article}

Keywords: single spin crossover molecules, spin states

Posted Date: March 16th, 2021

DOI: https://doi.org/10.21203/rs.3.rs-251042/v1

License: (c) (1) This work is licensed under a Creative Commons Attribution 4.0 International License. Read Full License 



\title{
1 Addressing single spin-crossover molecules in a two dimensional
}

monolayer

\author{
Yongfeng Tong, ${ }^{1}$ Massine Kelaï, ${ }^{1}$ Kaushik Bairagi, ${ }^{1,}{ }^{*}$ Vincent Repain, ${ }^{1}$ \\ Jérôme Lagoute, ${ }^{1}$ Yann Girard, ${ }^{1}$ Sylvie Rousset, ${ }^{1}$ Marie-Laure \\ Boillot, ${ }^{2}$ Talal Mallah, ${ }^{2}$ Cristian Enachescu, ${ }^{3}$ and Amandine Bellec ${ }^{1, \dagger}$ \\ ${ }^{1}$ Université de Paris, Laboratoire Matériaux et \\ Phénomènes Quantiques, CNRS, F-75013, Paris, France \\ ${ }^{2}$ Institut de Chimie Moléculaire et des Matériaux d'Orsay, \\ Université Paris-Saclay, CNRS, UMR 8182, 91405 Orsay 12 Cedex, France \\ ${ }^{3}$ Faculty of Physics, Alexandru Ioan Cuza University of Iasi, Iasi 700506, Romania
}

\begin{abstract}
Bistable spin-crossover molecules are particularly interesting to the development of innovative electronic and spintronic devices as they present two spin states that can be controlled by external stimuli. In this purpose, being able to switch at will the spin state of a single molecule in a dense molecular array is a key milestone. However, the elastic interactions between the molecules favour more cooperative behaviour where patches of neighbouring molecules switches simultaneously. We demonstrate here that the interaction of iron II spin-crossover molecules with a metallic substrate can strongly reduce their cooperative behaviour until addressing independently single molecular spin state. Mechanoelastic model is able to reproduce well such findings.
\end{abstract}

\footnotetext{
* present address: Université Grenoble Alpes, CEA, CNRS, Grenoble INP, IRIG-SPINTEC, 38054 Grenoble, France

† amandine.bellec@univ-paris-diderot.fr
} 
$11 \quad$ Molecular electronics and spintronics aim at using molecules as elementary components ${ }_{12}[1-3]$. In particular, switchable single molecules [4-8] are attracting lot of interest for ap${ }_{13}$ plications such as memory devices. In this purpose, spin-crossover molecules can be viewed 14 as promising molecules as they present two spin states which can be addressed by external 15 stimuli such as temperature or light [9-11]. Since the discovery of compounds showing a 16 first order thermal transition [12], the possibility to use spin-crossover molecules as mem17 ory has been investigated [13-15]. In bulk material, the key parameter to promote abrupt 18 thermal transition is the short and long elastic interactions inducing cooperativity among ${ }_{19}$ the molecules [16]. The size decrease, mandatory to incorporate such molecules in electronic 20 devices, completely modifies the cooperativity as demonstrated for nanoparticules [17-19] 21 and thin films [20,21]. Interestingly, at the molecular scale, the concept of memristance 22 has been demonstrated for isolated single spin-crossover molecules adsorbed on a substrate $23[22,23]$. This has been made possible because the molecules were decoupled from the metal24 lic layer. Indeed, in direct contact with a metallic substrate, the molecule's spin state is 25 usually locked without possibility to modify it [24].

26 The idea of addressing single molecules inside a self-assembled layer implies that the ${ }_{27}$ cooperativity have to be reduced so that the molecules are essentially independent from ${ }_{28}$ each other. Here we focus our study on the interplay between cooperativity and molecule29 substrate interaction along with their impact on the bistability of single spin-crossover so molecules self-organized in monolayer islands adsorbed on metallic substrates, namely ${ }_{31} \mathrm{Au}(111)$ and $\mathrm{Cu}(111)$. We demonstrate by scanning tunneling microscopy measurements the 32 possibility to address a single molecule inside a self-assembled molecular layer in direct con33 tact with a metal. These experimental results are supported by mechanoelastic simulations 34 that highlight the influence of the molecule-substrate interaction on the correlation between 35 the molecules as well as on the cluster relaxation and consequently on the cooperativity 36 inside the molecular layer.

37 The two dimensional molecular layers grown on metallic substrates are composed of ${ }_{38}\left[\mathrm{Fe}^{\mathrm{II}}\left(\left(3,5-\left(\mathrm{CH}_{3}\right)_{2} \mathrm{Pz}\right)_{3} \mathrm{BH}\right)_{2}\right](\mathrm{Pz}=$ pyrazolyl $)$ spin-crossover molecules, called FeMPz in the 39 following, which have a hysteretic thermal transition at a temperature around $190 \mathrm{~K}$ in 40 bulk [25]. On both $\mathrm{Au}(111)$ and $\mathrm{Cu}(111)$ substrates, the molecules FeMPz self-assembled ${ }_{41}$ in a molecular 2D network (see SI for details) [26, 27]. Figure 1.a presents a typical STM 42 topographic image on $\mathrm{Cu}(111)$ of the thermal state which corresponds to the state at low 

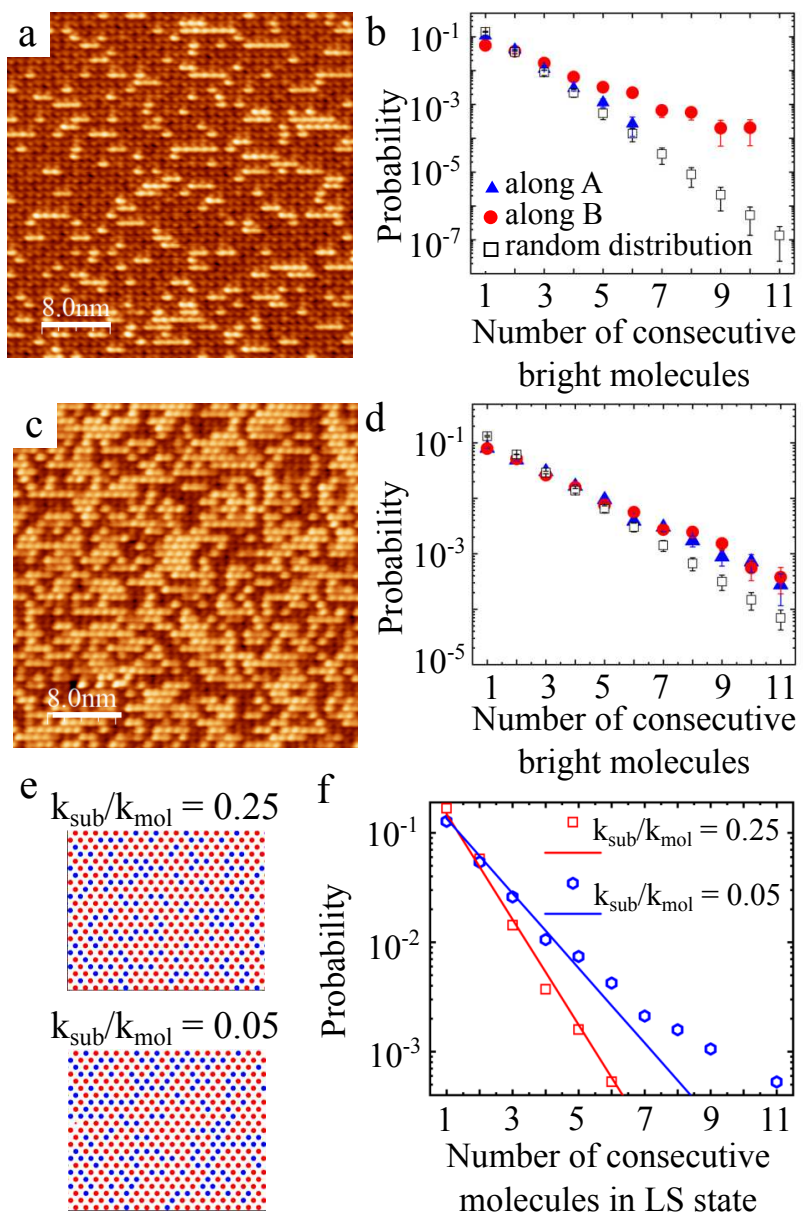

FIG. 1. Cooperativity in the molecular network on $\mathrm{Cu}(111)$ for the thermal and photoexcited states. a) $40 \times 40 \mathrm{~nm}^{2} \mathrm{STM}$ topographic image of the thermal state at $V=0.3 \mathrm{~V}$ and $I=5 \mathrm{pA}$. b) Probability to have $n$ consecutive bright molecules in the thermal state measured along $\vec{A}$ (blue triangles) and $\vec{B}$ (red dots) and compared to a random distribution (empty squares). c) $40 \times 40 \mathrm{~nm}^{2}$ STM topographic image of the photoexcited state at $V=0.3 \mathrm{~V}$ and $I=5 \mathrm{pA}$. d) Probability to have $n$ consecutive bright molecules in the photoexcited state measured along $\vec{A}$ (blue triangles) and $\vec{B}$ (red dots) and compared to a random distribution (empty squares). e) Snapshots of the thermal state for $k_{m o l}=4 \mathrm{~N} \cdot \mathrm{m}^{-1}$ and $k_{\text {sub }}=1 \mathrm{~N} \cdot \mathrm{m}^{-1}$ (top) and $k_{\text {sub }}=0.2 \mathrm{~N} \cdot \mathrm{m}^{-1}$ (bottom). The molecules in LS (HS) state are coded in blue (red). f) Probabilities to have $n$ consecutive molecules in LS state in red for $k_{\text {sub }}=1 \mathrm{~N} \cdot \mathrm{m}^{-1}$ and in blue for $k_{\text {sub }}=0.2 \mathrm{~N} \cdot \mathrm{m}^{-1}$. The probabilities obtained from the mechanoelastic model (symbols) are compared to a random distribution (lines). 
${ }_{43}$ temperature after the thermal transition. This state is measured at $4.6 \mathrm{~K}$ and at a bias 44 voltage of $0.3 \mathrm{~V}$ just after cooling down the sample from room temperature (tip approach 45 and scanning at $0.3 \mathrm{~V}$ and $50 \mathrm{pA}$ to avoid any switching of the molecules, see below). ${ }_{46}$ The molecules in the network appear either bright or dark which indicates the co-existence 47 of molecules with different electronic properties. The proportion of bright molecules $\left(p_{b}\right)$ 48 measured for the thermal state is $0.25 \pm 0.03$ (average over around 12500 molecules counted 49 in five STM images). The comparison with x-ray absorption spectroscopy measurements [28] 50 indicates that the bright molecules at $0.3 \mathrm{~V}$ are in the low spin (LS) state while the dark ones ${ }_{51}$ are in the high spin (HS) state similarly to what is observed on $\mathrm{Au}(111)$ (see Figure S2 in ${ }_{52} \mathrm{SI}$ ). The main difference between the $\mathrm{Cu}$ and Au substrates is the organization of the bright 53 molecules at $0.3 \mathrm{~V}$. Indeed, on $\mathrm{Au}(111)$ a superstructure is observed over a large area (up to ${ }_{54} 100 \times 100 \mathrm{~nm}^{2}$ ) [26] indicating strong interactions between the molecular spin states. For the ${ }_{55} \mathrm{Cu}(111)$, the distribution of the bright molecules seems more random (compare Figure 1.a 56 and Figure S2). To have a better understanding of the bright molecule distribution, we ${ }_{57}$ measured the probability of observed $n$ consecutive bright molecules along $\vec{A}$ (blue triangles) 58 and along $\vec{B}$ (red points) as reported in Figure 1.b. The measured probabilities are compared ${ }_{59}$ to the probability, given by $p_{b}^{n}\left(1-p_{b}\right)^{2}$, to have $n$ consecutive bright molecules for a random ${ }_{60}$ distribution of bright molecules. As one can see, the measured probabilities are larger than 61 what is expected for a random distribution especially along the direction $\vec{B}$. This indicates 62 that, when adsorbed on $\mathrm{Cu}(111)$, correlations exist between the molecules even though they ${ }_{63}$ are weaker than on $\mathrm{Au}(111)$.

${ }_{64}$ We investigate the photoexcitation at the molecular scale under blue light. Figure 1.c ${ }_{65}$ presents a typical STM topographic image obtained under blue light after saturation, i.e. ${ }_{66}$ no more evolution is measured. One can see that more molecules are appearing bright than 67 in the thermal state. The maximum conversion from the high spin state to the low spin 68 state, i.e. from dark to bright molecules at a bias voltage of $0.3 \mathrm{~V}$, leads to an increase ${ }_{69}$ of $p_{b}$ to $0.47 \pm 0.02$ (average over around 12500 molecules counted in five STM images). ${ }_{70}$ Once again, this proportion of bright molecules is consistent with the proportion of LS ${ }_{71}$ molecules measured by XAS [28]. For the photoexcited state, the probabilities to find $n$ 72 consecutive bright molecules along $\vec{A}$ and $\vec{B}$ are only slightly higher than for a random 73 distribution (Figure 1.d). The photoexcitation, happening randomly, seems to suppress the 74 local correlations. Qualitatively, the photoexcitation on both substrates can be compared. 
${ }_{75}$ For the $\mathrm{Au}(111)$ substrate, internal dynamics was observed during the photoexcitation which 76 we suspected to come from elastic interactions inside the molecular layer [26]. In contrast, 77 on the $\mathrm{Cu}(111)$ substrate, the STM images seem less noisy with an increase of the bright 78 molecule number and very few reverse transition (from bright to dark) as can be seen in the 79 film in SI. This qualitative comparison once again underlines the fact that the interaction 80 between the molecules are modified depending on the metallic substrate used and that the ${ }_{81}$ correlations are far larger in the molecular film grown on $\mathrm{Au}(111)$ than on $\mathrm{Cu}(111)$.

82 Using mechanoelastic simulations [29-31], we investigate the influence of the molecule83 susbtrate interaction on the local correlations. In this purpose, we fixed a value for the 84 intermolecular interaction $\left(k_{m o l}\right)$ to $4 \mathrm{~N}^{-1}{ }^{-1}$ and calculated the thermal state for two dif${ }_{85}$ ferent molecule-substrate interactions $\left(k_{\text {sub }}=0.2\right.$ and $\left.1 \mathrm{~N} \cdot \mathrm{m}^{-1}\right)$, which are represented in ${ }_{86}$ Figure 1.e. The first consequence of the $k_{\text {sub }}$ variation is the proportion in the thermal 87 state of molecules in low and high spin states, as previously reported [27]. We obtained 88 proportions of molecules in low spin state $\left(p_{L S}\right)$ of 0.46 and 0.34 for $k_{s u b}$ values of 0.2 and ${ }_{89} 1 \mathrm{~N} . \mathrm{m}^{-1}$, respectively. As presented in Figure 1.f, we then determined in the thermal state 90 the probability to have $n$ consecutive molecules in low spin state along the three equivalent ${ }_{91}$ directions of the triangular lattice. It appears that for a $k_{\text {sub }}$ value of $1 \mathrm{~N}^{-1} \mathrm{~m}^{-1}$ the probabil92 ity to have $n$ consecutive molecules in low spin state follows a random distribution (lines in

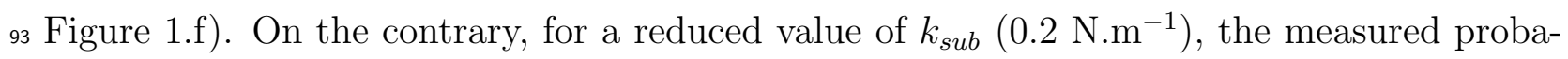
94 bility is away from the random distribution. Correlation between the molecules can also be ${ }_{95}$ quantified through the Moran index which decreases while the interaction to the substrate 96 increases (see Figure S3 in SI). Thus, increasing the interaction between the molecules and 97 the substrate leads to the decrease of the correlations inside the molecular layer. Once again ${ }_{98}$ this suggests that the molecules interact more with the $\mathrm{Cu}(111)$ substrate than with the $99 \mathrm{Au}(111)$.

100 Voltage pulses can also be used to trigger the transition of a single molecule from one 101 spin state to the other $[22,23,32]$. The manipulation with voltage pulses has thus been 102 tested on both metallic substrates. Figure 2.a and 2.b presents two examples of voltage ${ }_{103}$ pulses applied on molecules adsorbed on $\mathrm{Au}(111)$. For the first example, a series of four 104 consecutive pulses of $0.6 \mathrm{~V}$ (for $10 \mathrm{~ms}$ ) is applied on four nearby molecules - two bright 105 and two dark at $0.3 \mathrm{~V}$ as schematized by the black points in Figure 2.a. Interestingly, as ${ }_{106}$ can be seen in the STM topographic image recorded after the voltage pulse application, the 

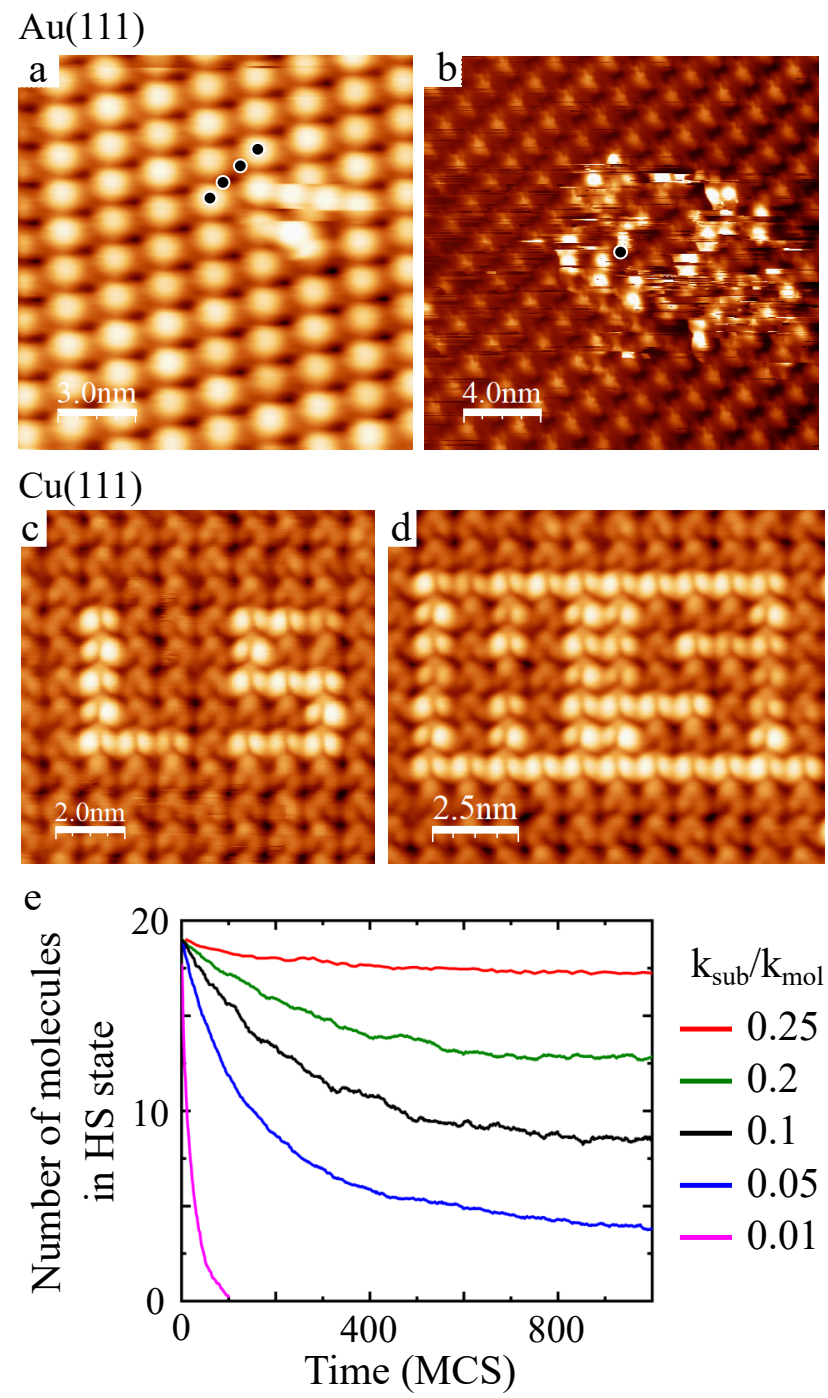

FIG. 2. Induced switching by voltage pulses. a) $15 \times 15 \mathrm{~nm}^{2}$ STM topographic image acquired after the application of four voltage pulses at $0.6 \mathrm{~V}$ on the different application points marked by the black dots $(V=0.3 \mathrm{~V}, I=20 \mathrm{pA}$, z varies from 0 to $1.7 \AA)$. b) $20 \times 20 \mathrm{~nm}^{2} \mathrm{STM}$ current image after a voltage pulse at $2.2 \mathrm{~V}(10 \mathrm{~ms})$ applied at the location of the black $\operatorname{dot}(V=0.3 \mathrm{~V},\langle I\rangle=10 \mathrm{pA}$, I varies from 0 to $250 \mathrm{pA}$ ). c) $10 \times 10 \mathrm{~nm}^{2}$ and d) $10 \times 12.5 \mathrm{~nm}^{2}$ topographic images where "LS" and "HS" have been written by voltage pulses $(V=0.3 \mathrm{~V}, I=3 \mathrm{pA}$, z varies from 0 to $2.63 \AA$ ). e) Relaxation of a cluster of molecules in HS state in an environment of molecules in LS state as a function of time (in Monte Carlo steps) for different ratio of $k_{s u b} / k_{m o l}$ with $k_{m o l}=4 \mathrm{~N} . \mathrm{m}^{-1}$.

107 molecules on which the pulses have been applied are still in their initial state while a defect 108 of bright molecules has appeared nearby, evidencing a non-local switching process [32]. The 
109 4-bright-molecule defect is stabilized for a few minutes (typically two minutes) before the 110 network recovers its initial superstructure (see Figure S4 in SI). Applying larger voltage 111 pulse can lead to the formation of larger defective area as presented in Figure S4 (see SI), 112 where lines of four or seven bright molecules are observed. Most probably, these 4-bright113 molecule and 7-bright-molecule lines can be stabilized because of their commensurability 114 with the thermal state superstructure [26]. Another example of voltage pulse is given in ${ }_{115}$ Figure 2.b. The voltage pulse of $2.2 \mathrm{~V}$ applied in the middle of the image induces the local 116 creation of a phase-shifted domain. As visible in Figure S5, the inner domain shrinks within 117 a few scans (less than fifteen minutes). Once again the created defect, here a phase-shifted 118 domain, is quickly healed. All these examples clearly show that applying voltage pulses 119 enables the conversion of some molecules from the HS state to the LS one (dark to bright) 120 but the elastic interactions within the 2D network (cooperativity) prevents addressing a ${ }_{121}$ single molecule and keeping the created defects or phase-shifted domains on $\mathrm{Au}(111)$.

${ }_{122}$ On the contrary, on $\mathrm{Cu}(111)$, we demonstrated the possibility to address the molecules ${ }_{123}$ one by one in the 2D network. Figures 2.c and 2.d present STM images where first the 124 molecules have been switched to the dark state, then one by one some molecules have been 125 switched back to the bright state to write HS and LS (the switching procedure is discussed 126 below). These features have been scanned for thirteen hours at a bias voltage of $0.3 \mathrm{~V}$ and 127 as can be seen in Figure S6 they remain intact with only few visible defects.

${ }_{128}$ To explain the role of the substrate on the stability of the switched molecules, we per129 formed a second mechanoelastic simulation. The initial state consists in a cluster of nineteen 130 molecules in the HS state surrounded by molecules in LS state. The relaxation of the HS 131 molecules in the cluster is simulated for different $k_{s u b} / k_{m o l}$ ratios for a given $k_{m o l}$ of $4 \mathrm{~N} . \mathrm{m}^{-1}$. ${ }_{132}$ The relaxation curves in Figure 2.e are obtained by averaging 100 relaxation curves (see ${ }_{133}$ Figure S7 in SI). The typical relaxation time for the cluster increases as the value of $k_{\text {sub }}$ in134 creases. This indicates that the spin state of the molecules can be stabilized by the substrate 135 interaction. This is in very good agreement with our observation that a switched state is 136 more stable on $\mathrm{Cu}(111)$ than on $\mathrm{Au}(111)$.

${ }_{137}$ We then investigated in more details the tunneling conditions to switch the molecules 138 from one spin state to the other on $\mathrm{Cu}(111)$. The first observation was realized by scanning 139 a photoexcited area at $0.7 \mathrm{~V}$. We observed by zooming out that in the area scanned at ${ }_{140} 0.7 \mathrm{~V}$ all the molecules are now appearing black at $0.3 \mathrm{~V}$ with clear-cut edges (see Fig. S8 

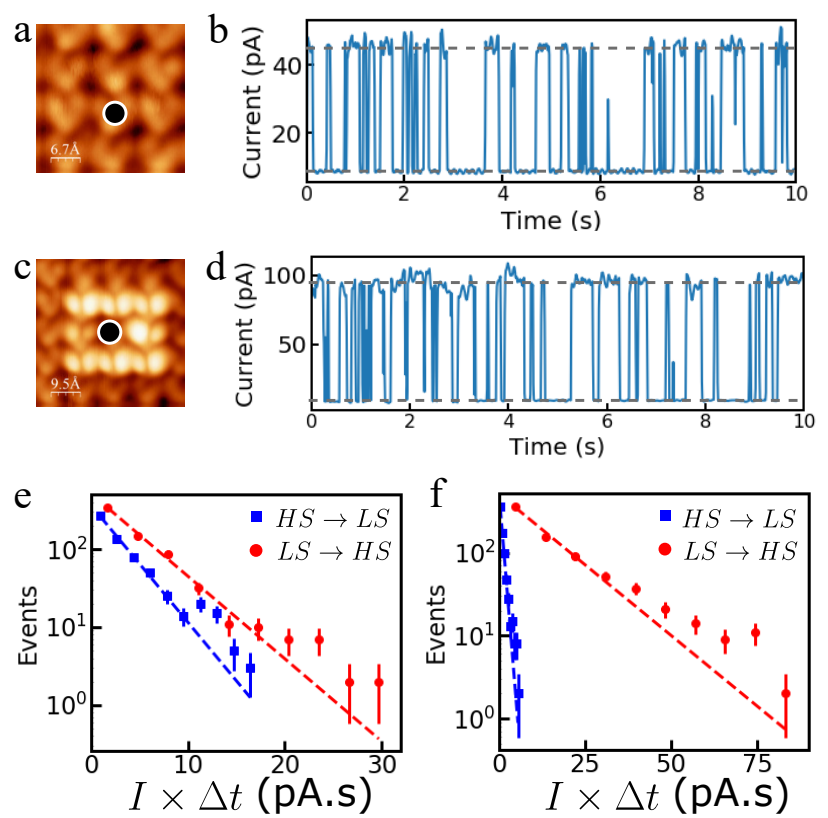

FIG. 3. Voltage pulses on $\mathrm{Cu}(111)$. a) $3.5 \times 3.5 \mathrm{~nm}^{2}$ STM topographic image of the initial state with dark (HS) neighbours $(V=0.3 \mathrm{~V}, I=5 \mathrm{pA})$ and c) $4.8 \times 4.8 \mathrm{~nm}^{2} \mathrm{STM}$ topographic image of the initial state with bright (LS) neighbours $(V=0.3 \mathrm{~V}, I=5 \mathrm{pA})$. The black dots indicate the position of the voltage pulses in both environments. b), d) Typical $I(t)$-traces recorded during voltage pulses of $0.5 \mathrm{~V}$ for dark (HS) and bright (LS) neighbours, respectively. e), f) Distribution of current times duration $(I \times \Delta t)$ of each plateau of the $I(t)$-traces for dark (HS) and bright (LS) neighbours, respectively. The red circles (blue squares) correspond to the experimental distribution of events from bright (LS) to dark (HS) (dark (HS) to bright (LS)) molecules. The dotted lines correspond to mono-exponential fits of each distribution.

${ }_{141}$ in SI). Thus, for this voltage bias the molecular switching from the LS state to the HS 142 state is favoured. The reverse switching can also be induced by using lower bias values. To 143 investigate in more details the possibility to locally induce the switching of the molecules at 144 positive voltage along with the influence of their first neighbours, we considered two initial 145 situations: either a molecule surrounded by dark (HS) molecules (Figure 3.a) or a molecule 146 surrounded by bright (LS) molecules (Figure 3.c). For both cases, it exists a threshold 147 voltage of $0.45 \mathrm{~V}$ below which no switching is observed within the temporal window of a 148 few minutes. Above this threshold, the switching can be induced. During voltage pulses of ${ }_{149} 0.5 \mathrm{~V}$, the tunneling current has been recorded as a function of time and typical $I(t)$-traces 
150 are presented in Figures 3.b and 3.d for dark (HS) or bright (LS) neighbours, respectively. ${ }_{151}$ From the analysis of the $I(t)$-traces, it is possible to extract a yield for the switching from 152 the HS state (dark) to the LS state (bright) $\left(Y_{H S \rightarrow L S}\right)$ and from the LS state (bright) to 153 the HS state (dark) $\left(Y_{L S \rightarrow H S}\right)$ for both environments. The yield is defined as $Y=\frac{e}{I \tau}$, 154 with $e$ the electron charge, $I$ and $\tau$ the characteristic current and time for each switching 155 extracted from the distribution of the current times duration (see Figures 3.e and 3.f and 156 SI ). When surrounded by molecules in the HS state, both switching events (from HS to LS 157 and LS to HS) are equally probable with equivalent yield values of $Y_{H S \rightarrow L S}=5.510^{-8}$ and ${ }_{158} Y_{L S \rightarrow H S}=3.910^{-8}$. On the contrary, when surrounded by molecules in the LS state, the 159 switching yield from HS-to-LS $\left(Y_{H S \rightarrow L S}=18.610^{-8}\right)$ is one order of magnitude larger than 160 the switching yield from LS-to-HS $\left(Y_{L S \rightarrow H S}=1.310^{-8}\right)$. Often, the final state imaged after 161 the voltage pulse reveals that some of the bright neighbours are switched to the dark state. ${ }_{162}$ Nonetheless, this clearly underlines that if the molecules can be addressed individually, the 163 neighbourhood is still influencing the switching properties. For higher bias voltage values, 164 the current traces are more noisy and the HS state is favoured as evidenced by the possibility 165 to create 'dark' area. Thus, on $\mathrm{Cu}(111)$ substrate, molecules can be individually addressed 166 thanks to the molecule-substrate interaction. The cooperativity is only reduced as evidenced 167 by the modification of the switching properties of a given molecule by its first neighbour's 168 spin state.

169 The different experiments and simulations described here highlight the role of the sub170 strate nature on the local correlation in the molecular layers and the possibility to stabilize 171 at will a molecule in a given spin state. The molecular layer on $\mathrm{Au}(111)$ presents a strong 172 cooperativity as evidenced by the long range correlation in the superstructure and the im173 possibility to control or stabilize any specific feature. $\mathrm{On} \mathrm{Cu}(111)$, the stronger molecule174 Substrate interaction weakens the cooperativity in the molecular layer, thus enabling to 175 address and stabilize the spin state of single molecules. The possibility to address single 176 molecule in a dense and organized network is interesting for future memory devices at the 177 molecular scale. 


\section{Experimental methods}

180 Prior to the molecular deposition, the metallic $\mathrm{Cu}(111)$ and $\mathrm{Au}(111)$ single crystals are 181 cleaned under ultra high vacuum (UHV, base pressure of $10^{-10} \mathrm{mbar}$ ) by sputtering $\left(\mathrm{Ar}^{+}\right.$ 182 ions at $600 \mathrm{eV}$ or $900 \mathrm{eV}$ for $\mathrm{Cu}(111)$ or $\mathrm{Au}(111)$ respectively) and annealing $\left(450^{\circ} \mathrm{C}\right.$ for 183 both substrates) cycles. Molecules FeMPz are then sublimated under UHV at a temperature ${ }_{184}$ of $85^{\circ} \mathrm{C}$ from a home-made Knudsen-cell-like evaporator on the metallic substrate. During 185 the molecular sublimation the single crystals are kept at low temperature (around $5 \mathrm{~K}$ ). ${ }_{186}$ The samples are then annealed at room temperature to enable the formation of the molec187 ular islands. All the measurements have been carried out on a low temperature scanning 188 tunneling microscope (STM, Omicron) operating at $4.6 \mathrm{~K}$. The light excitation has been 189 realized thanks to a super-continuum white laser followed by a filter (SuperK Evo/Varia, 190 NKTPhotonics) working at $405 \pm 5 \mathrm{~nm}$ with a power of $1.5 \mathrm{~mW}$ at the end of the optical 191 fibre before the UHV chamber.

\section{Mechanoelastic simulations}

For the mechanoelastic simulations, spin-crossover molecules are represented as balls 194 arranged in a triangular configuration and linked to their first neighbours situated in the 195 same layer by springs with an elastic constant $k_{m o l}$. The ball radii code for the molecular spin 196 states by taking a value of $0.22 \mathrm{~nm}$ and $0.2 \mathrm{~nm}$ for the HS and the LS states, respectively. ${ }_{197}$ The distance between two LS molecule centres is $1 \mathrm{~nm}$. The interaction to the substrate 198 is taken into account by linking each spin-crossover molecule to three surface molecules in 199 a face centred cubic configuration by springs with an elastic constant $k_{\text {sub }}$. The intrinsic

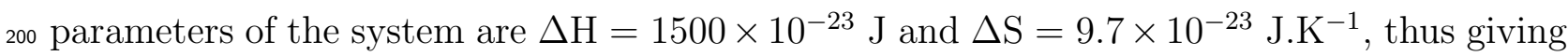
201 a thermal transition temperature around $155 \mathrm{~K}$.

\section{ACKNOWLEDGMENTS}

The authors thank the European Union's Horizon 2020 research and innovation program 204 under grant agreement $\mathrm{n}^{\circ} 766726$ for support. C.E. thanks UEFISCDI Romania (grant 
205 number PN-III-P4-ID-PCE-2020-1946).

229 [13] O. Kahn and J. Launay, Chemtronics 3, 140 (1988).

230 [14] O. Kahn, J. Kröber, and C. Jay, Advanced Materials 4, 718 (1992).

231 [15] O. Kahn and C. J. Martinez, Science 279, 44 (1998).

232 [16] K. Ridier, G. Molnár, L. Salmon, W. Nicolazzi, and A. Bousseksou, Solid State Sciences 74,

233

1] M. Cinchetti, V. A. Dediu, and L. E. Hueso, Nature Materials 16, 507 (2017).

[2] S. Delprat, M. Galbiati, S. Tatay, B. Quinard, C. Barraud, F. Petroff, P. Seneor, and R. Mattana, Journal of Physics D: Applied Physics 51, 473001 (2018).

[3] E. Coronado, Nature Reviews Materials 5, 87 (2019).

[4] H. Böckmann, S. Liu, J. Mielke, S. Gawinkowski, J. Waluk, L. Grill, M. Wolf, and T. Kumagai, Nano Letters 16, 1034 (2016).

5] C. Nacci, M. Baroncini, A. Credi, and L. Grill, Angewandte Chemie International Edition $\mathbf{5 7 ,} 15034(2018)$.

6] A. Köbke, F. Gutzeit, F. Röhricht, A. Schlimm, J. Grunwald, F. Tuczek, M. Studniarek, D. Longo, F. Choueikani, E. Otero, P. Ohresser, S. Rohlf, S. Johannsen, F. Diekmann, K. Rossnagel, A. Weismann, T. Jasper-Toennies, C. Näther, R. Herges, R. Berndt, and M. Gruber, Nature Nanotechnology 15, 18 (2020).

[7] R. Harsh, F. Joucken, C. Chacon, V. Repain, Y. Girard, A. Bellec, S. Rousset, R. Sporken, A. Smogunov, Y. J. Dappe, and J. Lagoute, The Journal of Physical Chemistry Letters 10, $6897(2019)$.

[8] M. Bouatou, C. Chacon, F. Joucken, Y. Girard, V. Repain, A. Bellec, S. Rousset, R. Sporken, C. González, Y. J. Dappe, and J. Lagoute, The Journal of Physical Chemistry C 124, 15639 (2020).

[9] J. A. Real, A. B. Gaspar, and M. C. Muñoz, Dalton Transactions , 2062 (2005).

[10] W. Nicolazzi and A. Bousseksou, Comptes Rendus Chimie 21, 1060 (2018).

[11] J. Dugay, M. Aarts, M. Gimz-Marqu T. Kozlova, H. W. Zandbergen, E. Coronado, and H. S. J. van der Zant, Nano Letters 17, 186 (2017).

2] W. A. Baker and H. M. Bobonich, Inorganic Chemistry 3, 1184 (1964). 
${ }_{234}^{2}$ [17] F. Volatron, L. Catala, E. Rivière, A. Gloter, O. Stéphan, and T. Mallah, Inorganic Chemistry $235 \quad \mathbf{4 7}, 6584(2008)$.

236 [18] G. Félix, W. Nicolazzi, L. Salmon, G. Molnár, M. Perrier, G. Maurin, J. Larionova, J. Long, 237 Y. Guari, and A. Bousseksou, Physical Review Letters 110 (2013).

238 [19] R. Bertoni, M. Lorenc, H. Cailleau, A. Tissot, J. Laisney, M.-L. Boillot, L. Stoleriu, A. Stancu, 239 C. Enachescu, and E. Collet, Nature Materials 16, 606 (2016).

$240[20]$ T. Groizard, N. Papior, B. Le Guennic, V. Robert, and M. Kepenekian, The Journal of $241 \quad$ Physical Chemistry Letters 8, 3415 (2017).

${ }_{242}^{2}$ [21] L. Kipgen, M. Bernien, S. Ossinger, F. Nickel, A. J. Britton, L. M. Arruda, H. Naggert, ${ }_{243}$ C. Luo, C. Lotze, H. Ryll, F. Radu, E. Schierle, E. Weschke, F. Tuczek, and W. Kuch, $244 \quad$ Nature Communications 9, 2984 (2018).

245 [22] T. Miyamachi, M. Gruber, V. Davesne, M. Bowen, S. Boukari, L. Joly, F. Scheurer, G. Rogez, T. K. Yamada, P. Ohresser, E. Beaurepaire, and W. Wulfhekel, Nature Communications 3, 938 (2012).

248 [23] T. Jasper-Tönnies, M. Gruber, S. Karan, H. Jacob, F. Tuczek, and R. Berndt, Nano Letters $249 \quad \mathbf{1 7}, 6613(2017)$.

250 [24] M. Gruber, V. Davesne, M. Bowen, S. Boukari, E. Beaurepaire, W. Wulfhekel, and T. Miya$251 \quad$ machi, Physical Review B 89, 195415 (2014).

252 [25] O. Iasco, M.-L. Boillot, A. Bellec, R. Guillot, E. Rivière, S. Mazerat, S. Nowak, D. Morineau,

256

257

$$
258
$$
A. Brosseau, F. Miserque, V. Repain, and T. Mallah, Journal of Materials Chemistry C 5, 11067 (2017).

[26] K. Bairagi, O. Iasco, A. Bellec, A. Kartsev, D. Li, J. Lagoute, C. Chacon, Y. Girard, S. Rousset, F. Miserque, Y. J. Dappe, A. Smogunov, C. Barreteau, M.-L. Boillot, T. Mallah, and V. Repain, Nature Communications 7, 12212 (2016).

58 [27] C. Fourmental, S. Mondal, R. Banerjee, A. Bellec, Y. Garreau, A. Coati, C. Chacon, Y. Girard, J. Lagoute, S. Rousset, M.-L. Boillot, T. Mallah, C. Enachescu, C. Barreteau, Y. J. Dappe, A. Smogunov, S. Narasimhan, and V. Repain, The Journal of Physical Chemistry Letters 10, $4103(2019)$.

[28] L. Zhang, Y. Tong, M. Kelai, A. Bellec, J. Lagoute, C. Chacon, Y. Girard, S. Rousset, M.L. Boillot, E. Rivière, T. Mallah, E. Otero, M.-A. Arrio, P. Sainctavit, and V. Repain, Angewandte Chemie International Edition 59, 13341 (2020). 
265 [29] C. Enachescu, L. Stoleriu, A. Stancu, and A. Hauser, Physical Review Letters 102, 257204 266 (2009), publisher: American Physical Society.

267 [30] C. Enachescu, M. Nishino, S. Miyashita, L. Stoleriu, and A. Stancu, Physical Review B 86, $268054114(2012)$.

269 [31] C. Enachescu and A. Hauser, Physical Chemistry Chemical Physics 18, 20591 (2016).

270 [32] T. G. Gopakumar, F. Matino, H. Naggert, A. Bannwarth, F. Tuczek, and R. Berndt, Ange$271 \quad$ wandte Chemie International Edition 51, 6262 (2012). 


\section{Figures}
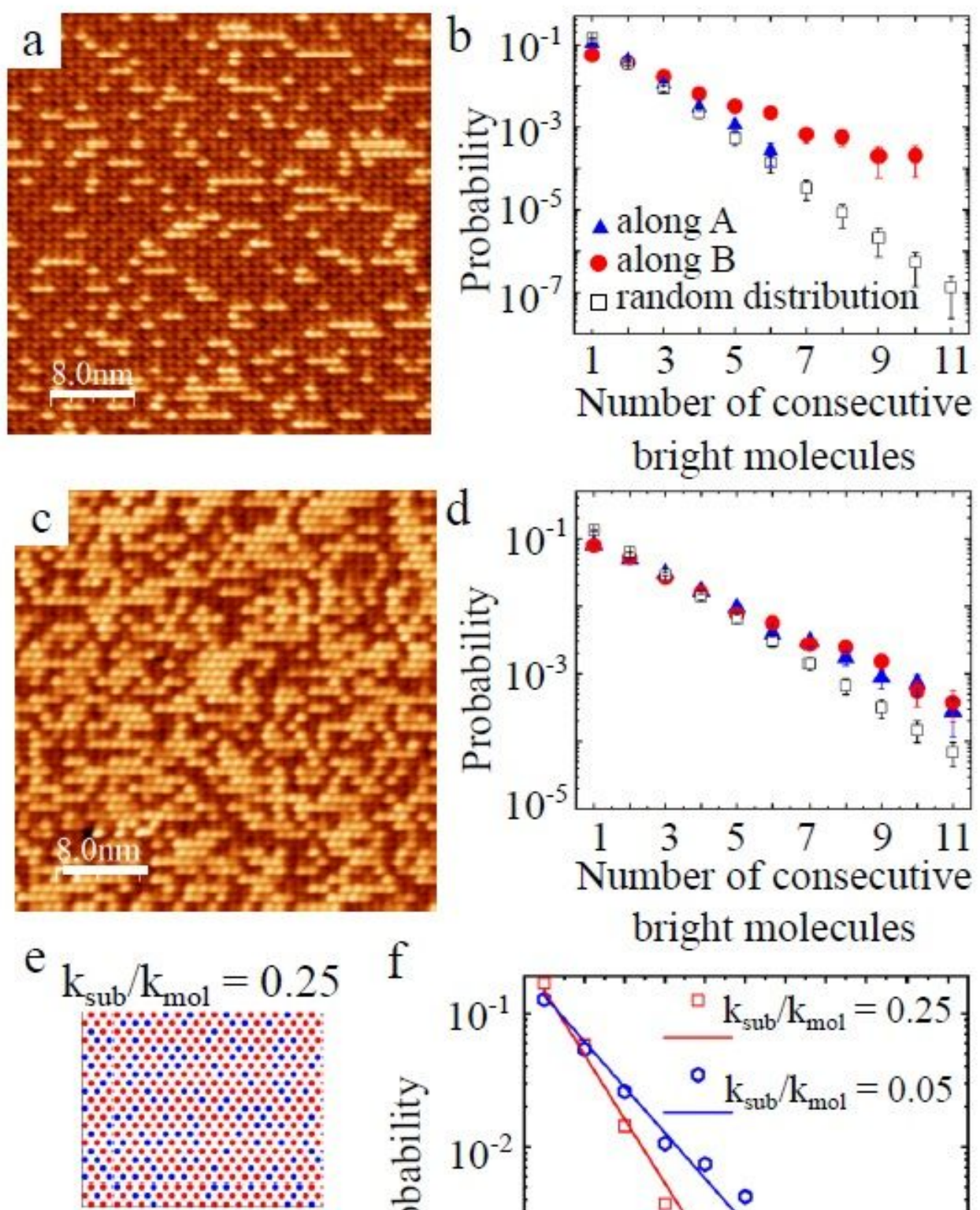

$\mathrm{k}_{\text {sub }} / \mathrm{k}_{\text {mol }}=0.05$

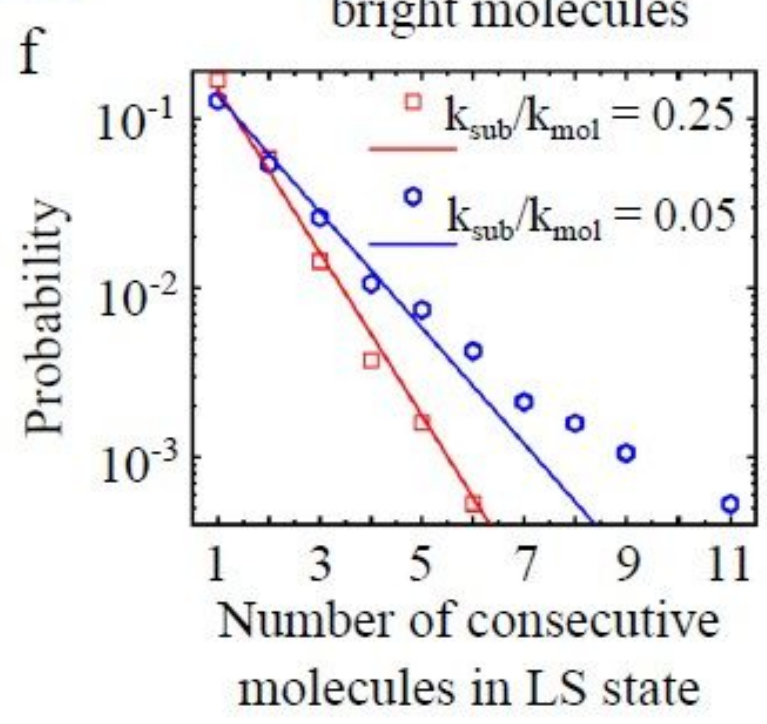

Figure 1

Cooperativity in the molecular network on $\mathrm{Cu}(111)$ for the thermal and photoexcited states. a) $40 \times 40$ $\mathrm{nm} 2$ STM topographic image of the thermal state at $\mathrm{V}=0: 3 \mathrm{~V}$ and $\mathrm{I}=5 \mathrm{pA}$. b) Probability to have $\mathrm{n}$ consecutive bright molecules in the thermal state measured along $\sim A$ (blue triangles) and $\sim B$ (red dots) 
and compared to a random distribution (empty squares). c) $40 \times 40 \mathrm{~nm} 2 \mathrm{STM}$ topographic image of the photoexcited state at $\mathrm{V}=0: 3 \mathrm{~V}$ and $\mathrm{I}=5 \mathrm{pA}$. d) Probability to have $\mathrm{n}$ consecutive bright molecules in the photoexcited state measured along $\sim \mathrm{A}$ (blue triangles) and $\sim \mathrm{B}$ (red dots) and compared to a random distribution (empty squares). e) Snapshots of the thermal state for kmol $=4 \mathrm{~N} \cdot \mathrm{m}-1$ and $k s u b=1 \mathrm{~N} \cdot \mathrm{m}-1$ (top) and ksub $=0.2 \mathrm{~N} . \mathrm{m}-1$ (bottom). The molecules in LS (HS) state are coded in blue (red). $\mathrm{f}$ ) Probabilities to have $\mathrm{n}$ consecutive molecules in LS state in red for ksub $=1 \mathrm{~N} \cdot \mathrm{m}-1$ and in blue for $\mathrm{ksub}=$ $0.2 \mathrm{~N} . \mathrm{m}-1$. The probabilities obtained from the mechanoelastic model (symbols) are compared to a random distribution (lines). 

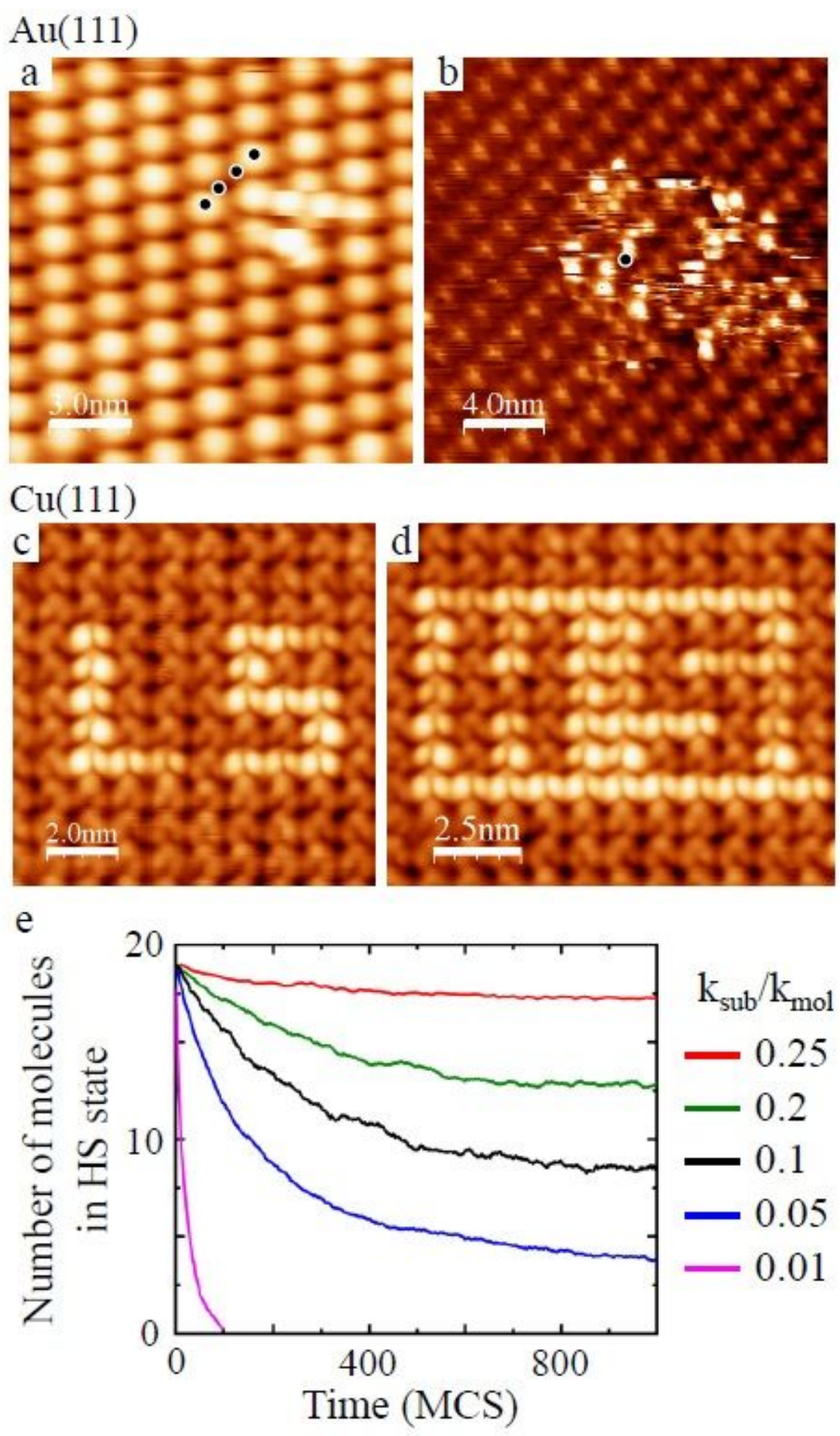

\section{Figure 2}

Induced switching by voltage pulses. a) $15 \times 15 \mathrm{~nm} 2$ STM topographic image acquired after the application of four voltage pulses at $0.6 \mathrm{~V}$ on the different application points marked by the black dots ( $\mathrm{V}$ $=0: 3 \mathrm{~V}, \mathrm{I}=20 \mathrm{pA}, \mathrm{z}$ varies from 0 to $1.7 \mathrm{~A}$ ). b) $20 \times 20 \mathrm{~nm} 2 \mathrm{STM}$ current image after a voltage pulse at 2.2 $\mathrm{V}(10 \mathrm{~ms})$ applied at the location of the black dot $(\mathrm{V}=0: 3 \mathrm{~V},<\mathrm{I}>=10 \mathrm{pA}$, I varies from 0 to $250 \mathrm{pA})$. c) 10 x $10 \mathrm{~nm} 2$ and d) $1012: 5 \mathrm{~nm} 2$ topographic images where $\backslash L S "$ and $\backslash H S "$ have been written by voltage 
pulses ( $\mathrm{V}=0: 3 \mathrm{~V}, \mathrm{I}=3 \mathrm{pA}, \mathrm{z}$ varies from 0 to $2.63 \mathrm{~A})$. e) Relaxation of a cluster of molecules in HS state in an environment of molecules in LS state as a function of time (in Monte Carlo steps) for different ratio of $\mathrm{ksub}=\mathrm{kmol}$ with $\mathrm{kmol}=4 \mathrm{~N} \cdot \mathrm{m}-1$.
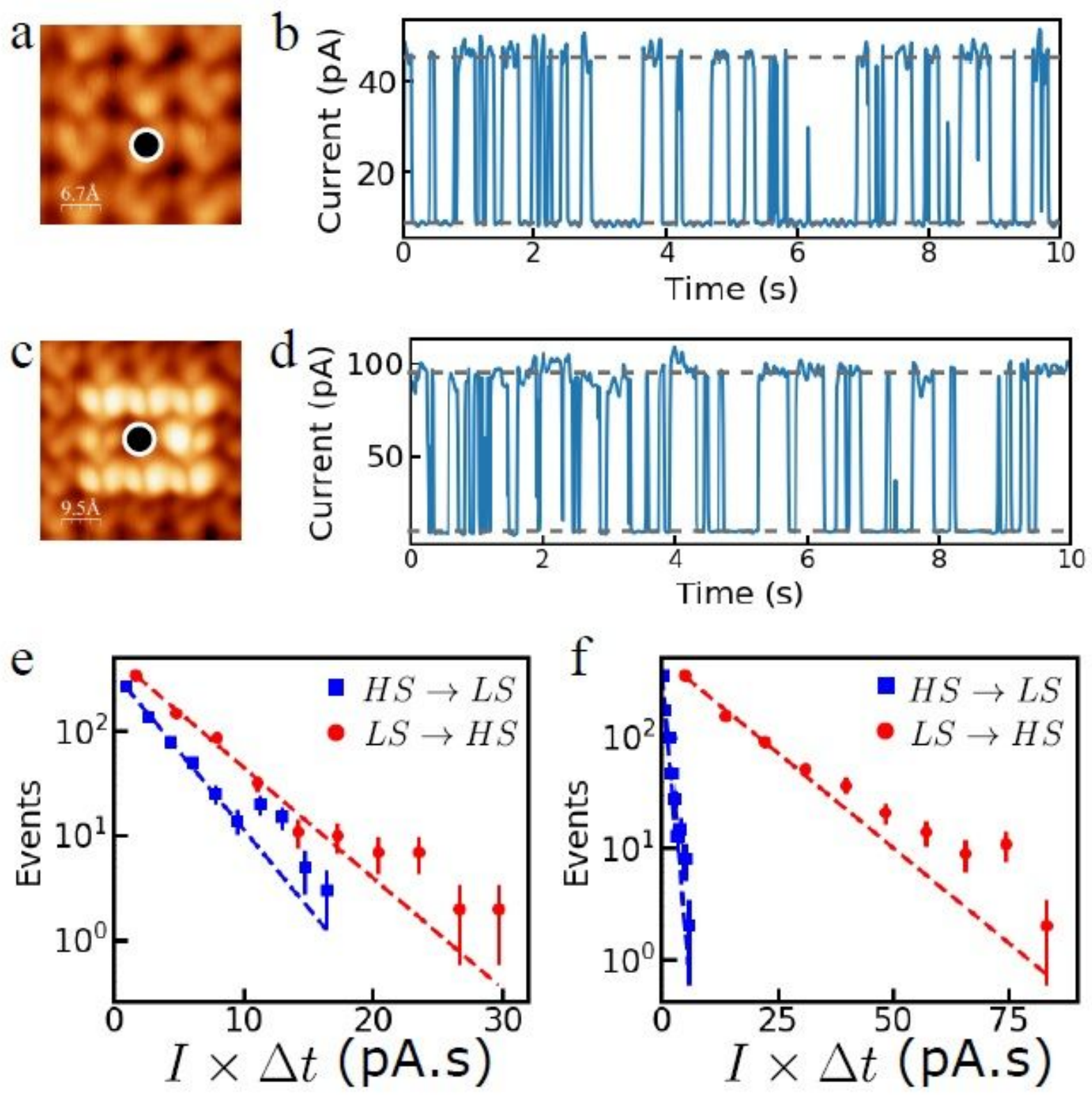

\section{Figure 3}

Voltage pulses on $\mathrm{Cu}(111)$. a) 3:5 $\times 3: 5 \mathrm{~nm} 2 \mathrm{STM}$ topographic image of the initial state with dark (HS) neighbours $(\mathrm{V}=0: 3 \mathrm{~V}, \mathrm{I}=5 \mathrm{pA})$ and c) 4:8 $\times 4: 8 \mathrm{~nm} 2 \mathrm{STM}$ topographic image of the initial state with bright (LS) neighbours ( $\mathrm{V}=0: 3 \mathrm{~V}, \mathrm{I}=5 \mathrm{pA})$. The black dots indicate the position of the voltage pulses in both environments. b), d) Typical I(t)-traces recorded during voltage pulses of $0.5 \mathrm{~V}$ for dark (HS) and bright (LS) neighbours, respectively. e), f) Distribution of current times duration (It) of each plateau of the 
I(t)-traces for dark (HS) and bright (LS) neighbours, respectively. The red circles (blue squares) correspond to the experimental distribution of events from bright (LS) to dark (HS) (dark (HS) to bright (LS)) molecules. The dotted lines correspond to mono-exponential ts of each distribution.

\section{Supplementary Files}

This is a list of supplementary files associated with this preprint. Click to download.

- Tongetalfilm.mp4

- TongetalSI.pdf 